\title{
Detrended Fluctuation Analysis of Acupuncture based on Neural Electrical Signals
}

\author{
Xiaobin Wang ${ }^{1}$, Yanqiu Che ${ }^{1}$, Chunxiao Han $^{1}{ }^{*}$,Yingmei Qin ${ }^{1}$ \\ ${ }^{1}$ Tianjin Key Laboratory of Information Sensing \& Intelligent Control \\ Tianjin University of Technology and Education \\ Tianjin, China \\ E-mail: cxhan@tju.edu.cn
}

\begin{abstract}
Manual acupuncture (MA) is a traditional Chinese Medicine clinical therapy. Although its effectiveness has been proved by clinical trials over thousands of years, the effect and mechanism of acupuncture on the nervous system is not yet clear.Brain is senior central nervous system, and acupuncture as an external stimulation, can induce various electrical activitiesin the brain cortex.In this paper, we design experiment that acupuncture at acupointZusanli withfour different frequencies, andcollect neural signalson the brain.Detrended fluctuation analysis method is used to extract features from EEG signals evoked by different MA manipulations, which may provide theoretical basis forquantifying acupuncture and revealing the mechanism of acupuncture from the aspect of neural electrical signals.
\end{abstract}

Keywords-Acupuncture; EEG; Detrended fluctuation analysis

\section{INTRODUCTION}

Acupuncture is a unique non-drug treatment method in medical field in China.Two thousand years of clinical practice has verified the effectiveness of acupuncture[13].Organism is taken into consideration as a whole, and acupuncture not only has a significant effect in the treatment of disease, but also can improve people's physical conditions.However, regulation mechanism of acupuncture is still not clear,which has seriously restricted the developmentof acupuncture in medical field.

When human is exposed on external stimuli,the relevant regions of the brain will appearcorresponding discharge phenomenon.Recent clinical and experimental data show thatacupuncture can induce specific patterns of brain activity, but the specific effects of acupuncture on the brain is still unknown.Therefore, acquire related EEG signals from the corresponding brain regions, extract features from EEG, so as to obtain the effect of acupuncture on the brain neural electrical activities, which provides certain theoretical basis for reveal the mechanism of acupuncture, and accelerates the process of modernization of traditional acupuncture in the medical field.

Zusanliis a widely used acupoint, which is located under the knee along the stomach meridian. A large number of ancient and modern clinicalpractices have proved that it is able to regulate and control a variety of diseases and physical

This work is supported by The Natural Science Foundation of Tianjin (Grant No. 13JCQNJC03700), The National Natural Science Foundation of China (Grant No. 61104032, 61401312). We would also acknowledge the support of Tianjin University of Technology and Education (Grant No. RC14-49 and RC14-59). fitness. According to the human anatomy structure of nervous system,when acupuncture at acupointZusanli, spinal dorsal root and dorsal horn will generate corresponding neural discharge, and neural information will be uploaded to the brain. Therefore, acupuncture has a certain impact on the brain, while brain will send control instructionsbased on the acupuncture and focus.

It has been shown that EEG signalsemerge long range correlation features of shock dynamics[4].Detrended fluctuation analysis (DFA)is proposed based on deoxyribonucleic acid (DNA) mechanism, which can describe the long range power-law correlation of nonstationary time series with the scaling exponent [5].It can identify underlying self-similarity from non-stationary time series, and avoid detecting pseudo correlation caused by noise and external trends. In recent years, it has been applied in life science, geology, meteorology and economic fields, whichbecomes an effective tool for non-stationary time series.DFAalso provides a quantitative description for feature extraction and pattern recognition of anesthesia, sleep and seizure by analyzing EEG signals [6-9].

In this paper, DFA method is used to analyzethe longrange exponential correlation of EEG signals evoked by acupuncture from the aspect ofdifferent brainrhythms. Through statistical analysis, extract the corresponding feature parameters, analysis the effect of acupuncture on the nervous system.

The paper is organized as follows.In section 2,the experimental process is interpreted.In section 3 , the analysis method is introduced.In section 4,data analysis results are presented. Finally,conclusions are given.

\section{EXPERIMENTS}

Subjects are 9 healthy volunteers ( 6 males and 3 females, aged between 23 and 27). During the experiments, environment should be quiet and dark. Subjects keep eyes closed in the whole experimental process, but stay awake.When there is no apparent EMG disturbance, physicians start needling.For each subject, the process lasts about 90 minutes. At first, let the subject relax until EEG signal becomes stable; Next, acupuncture at acupointZusanli for 2 minutes; then retain needle without manipulation for 10 
minutes break. Repeat this sub-process four times. All acupuncture manipulations are twirling reinforcing manipulation. Each sub-process uses acupuncture manipulation with different frequency, such as 50 times $/ \mathrm{min}$, 100 times/minute, 150 times/minute and 200 times/minute.The order of acupuncture manipulations is random, so as to eliminate the effect of the manipulation order. The acupuncture experimental process is shown in Fig. 1.

EEG signals are recorded continuously during the whole experimental process from 20 electrodes according to 10-20 system (Fp1(1), Fp1(2), F7(3), F3(4), Fz(5),F4(6), F8(7), T3(8), C3(9), Cz(10), C4(11), T4(12), T5(13), P3(14), $\mathrm{Pz}(15), \mathrm{P} 4(16), \mathrm{T} 6(17), \mathrm{O} 1(18), \mathrm{Oz}(19)$ and O2(20)). Sampling frequency is $256 \mathrm{~Hz}$. Hardware filter passband is $0.5 \mathrm{~Hz}-100 \mathrm{~Hz}$. For each subject, we intercept 6 epochs of data, which corresponds to six states, i.e. before acupuncture (BA), acupuncture with 50 times/min (A50), acupuncture with 100 times/min (A100), acupuncture with 150 times/min (A150), acupuncture with 200 times/min (A200) and after acupuncture (AA). Each epoch last 80s.

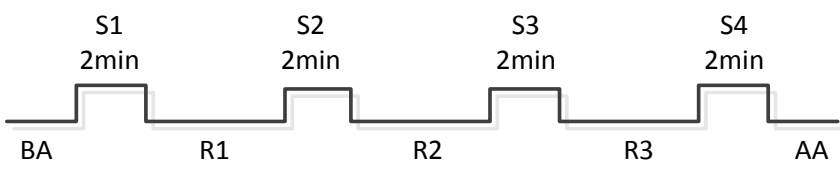

Fig. 1.Acupuncture experimental process. (BA - Before acupuncture; AA AfterAcupuncture; S1, S2, S3, S4 - different acupuncture manipulations; R1, R2, R3- Retaining needle between different acupuncture manipulations )

\section{METHODS}

\section{A. Detrended fluctuation analysis method, DFA}

Detrended fluctuation analysis method (DFA) is proposed based on stochastic process theory and chaos dynamics, which can describe the long-range correlation of non-stationary time series[5].DFAalgorithm is as follows [10-11].

Step 1: Calculatethe cumulative deviation of the original sequence $\{\mathrm{x}(t) \mid t=1,2, \ldots, N\}$. Then, $\mathrm{x}(t)$ is converted to a new sequence $\{\mathrm{y}(t) \mid t=1,2, \ldots, N\}$

$$
y(t)=\sum_{i=1}^{t}(x(i)-\bar{x})
$$

where $\bar{x}$ is the average value of $\mathrm{x}(t)$.

Step 2: $\{\mathrm{y}(t) \mid t=1,2, \ldots, N\}$ is divided into $m$ disjoint interval. $s$ is the interval length, which presents the time scale. $m=N / s$ is interval number.Fit local trendfunction $\mathrm{y}_{s}(t)$ of each subinterval with least squares method. According to the the degree of polynomial fitting, it can be written as firstorder detrended fluctuation analysis(DFA1),second-order detrended fluctuation analysis(DFA2)etc.
Step 3: Eliminate localtrend of sequence $y(t)$ at each interval,and calculate the root mean square of the new sequence, i.e. fluctuation function

$$
F(s)=\sqrt{\frac{1}{N} \sum_{i=1}^{N}\left(y(t)-y_{s}(t)\right)^{2}}(2)
$$

Step 4: For different intervallength $s$, repeat Step 3. Then, the relationship between $s$ and $F(s)$ is obtained.

$$
F(s) \propto s^{\alpha}
$$

Usually, $F(s)$ will increase with the increase of $s$. The linear relationship in double logarithmic diagram of $F(s)$ and $s$ represents long range correlation phenomenon.Scaling exponent $\alpha$ is the slope of linear fitting by using least square method, which represents long-range correlation property oftime series and is independent of signal amplitude and external trends.If $0<\alpha<0.5$, sequenceshows inverse correlation in the power-lawform.If $\alpha=0.5$, sequence is completely random and has no long-range correlation phenomenon. If $0.5<\alpha<1$, there is continuouscorrelation in the sequence. If $\alpha=1$, the sequence is $1 / \mathrm{f}$ noise.If $\alpha>1$, there isn't power-law formed correlation in the sequence. If $\alpha=1.5$, the sequence fluctuation is Brown motion, which shows long-range correlation.If $\alpha>1.5$, the sequence shows periodic fluctuation.

\section{B. Normalizationmethod}

In order to eliminate individual differences and improve the comparison of the results, we define changing rate as normalization index.

$$
R_{\alpha}=\frac{\alpha}{\alpha_{0}}
$$

where $\alpha_{0}$ is the scaling exponent calculated from EEG before acupuncture, which is used as reference value. $\alpha$ is the scaling exponent of EEG under different acupuncture epochs. $R_{\alpha}$ reflectsthe relative variance compared with $\alpha_{0}$. If $R_{\alpha}>1$, scaling exponent of current epoch increase compared with the epoch before acupuncture, vice versa.

\section{Statistical analysis}

One-wayanalysisofvariance (ANOVA)is introduced to identify the difference between epoch before acupuncture and other epochs, respectively.In this paper, $P<0.05$ indicates that there is difference between different epochs, whilethere is significant difference when $P<0.01$.

\section{RESUlTS}

DFA method is used to study the long-range correlation from EEG signals under different acupuncture epochs.For a certain epoch, we adopt sliding window technique with $4 \mathrm{~s}$ window length and 3s window overlap to calculate $\alpha$ of each channel. For each window, we get a $\alpha$. Hence, as the window sliding, there is a sequence of $\alpha$ for each channel, 
then the average of sequence $\alpha$ is scaling exponent of corresponding channel.

Fig. 2 shows relative scaling exponent of each channel under different acupuncture epochs at different EEG rhythms. The abscissa represents EEG channel number, and the vertical represents $R_{\alpha}$. Different line type means different epochs. Fig. 2(a) is scaling exponent of EEG signals at $0.5-47 \mathrm{~Hz}$, and Fig. 2(b)-(e) are scaling exponent at different EEG rhythm. It is found that compared with BA epoch, $R_{\alpha}$ increases at delta and theta rhythms for most brain areas under other five epochs, while decreases at alpha and beta rhythms for most brain areas.

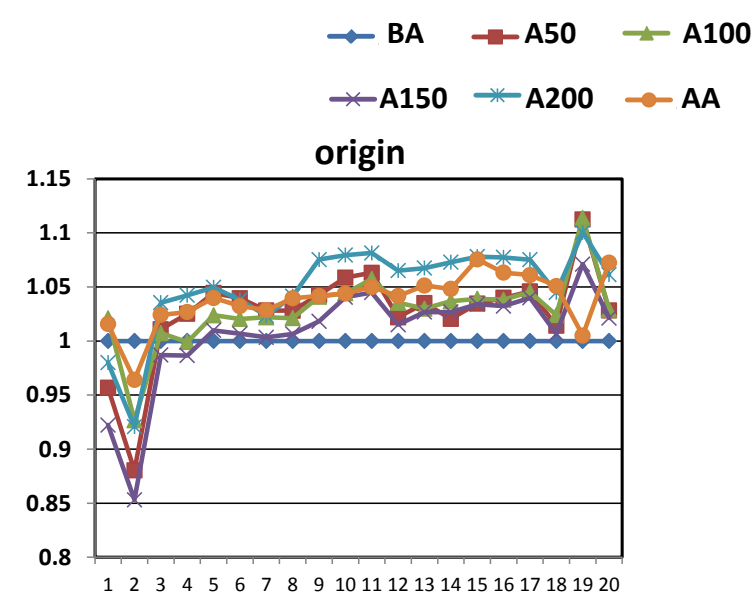

(a)

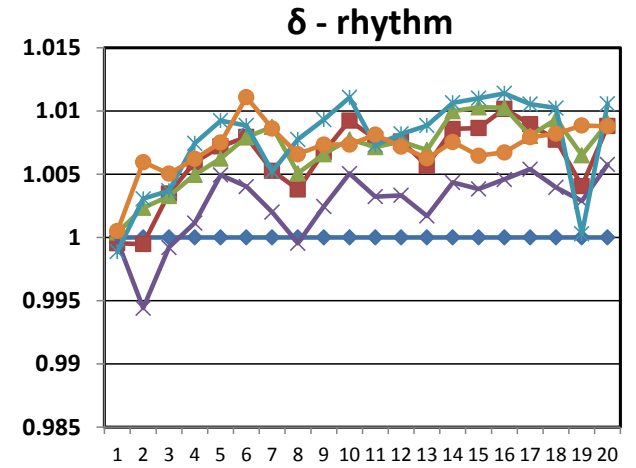

(b)

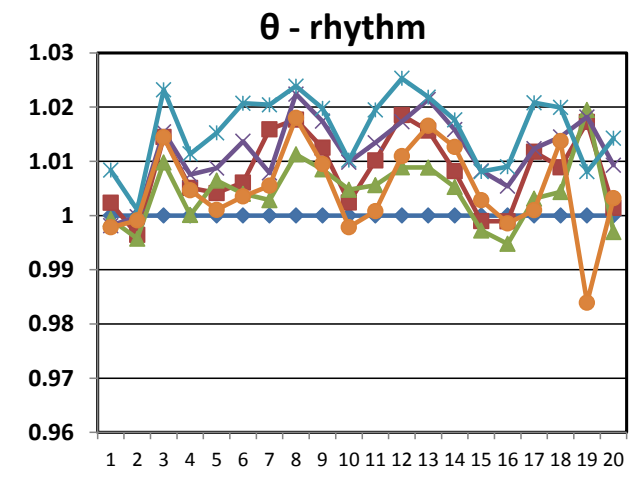

(c)

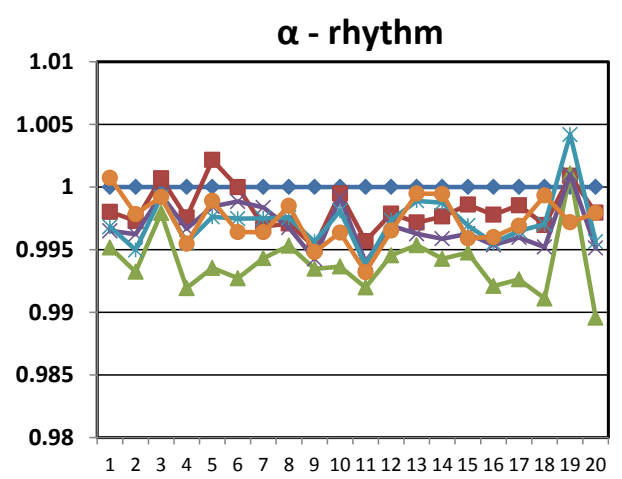

(d)

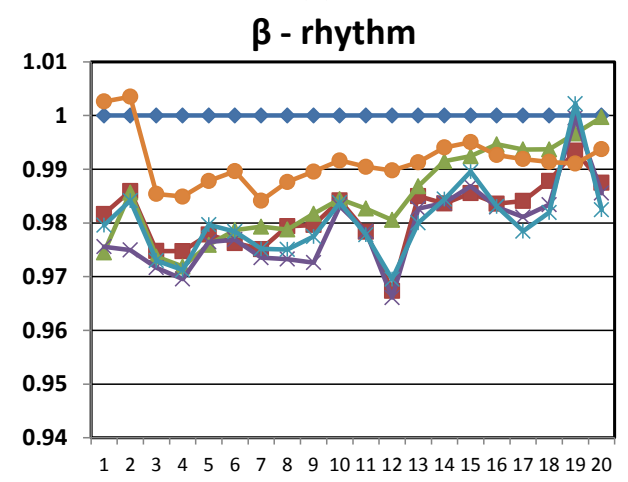

(e)

Fig.2.Normalized scaling exponent of 20 EEG channels under different acupunctureepochs at different EEG rhythm for nine subjects.

Fig.3is statistical analysis results of normalized scaling exponent of global brain domain under different acupuncture epochs at different EEG rhythm for nine subjects. It can be seen that (1) $R_{\alpha}$ during A50, A200 and AA epochs is much higher than that during BA epoch the delta rhythm; (2) $R_{\alpha}$ during A100 and A200 epochs increase compared with BA epoch at the theta rhythm; (3) $R_{\alpha}$ during A100 is much lower than that during BA epoch at the alpha rhythm; (4) $R_{\alpha}$ during A50, A150 and A200 epochs decrease compared with BA epoch at the beta rhythm.

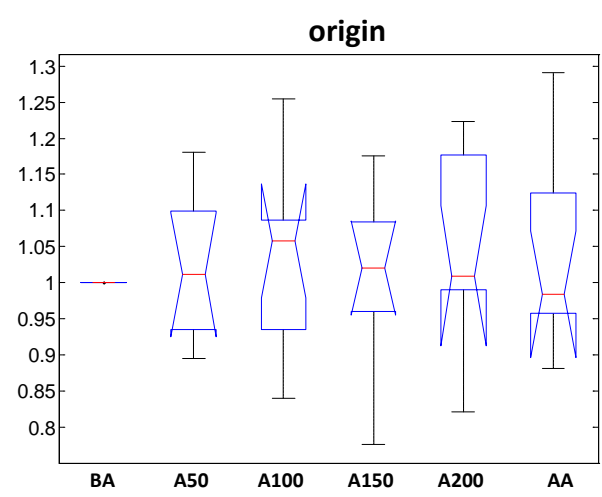

(a) 


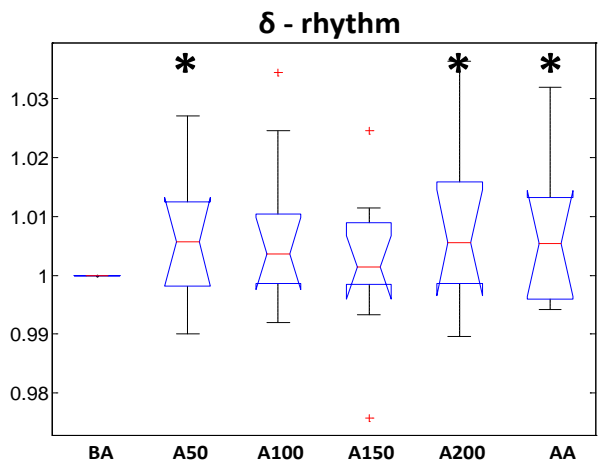

(b)

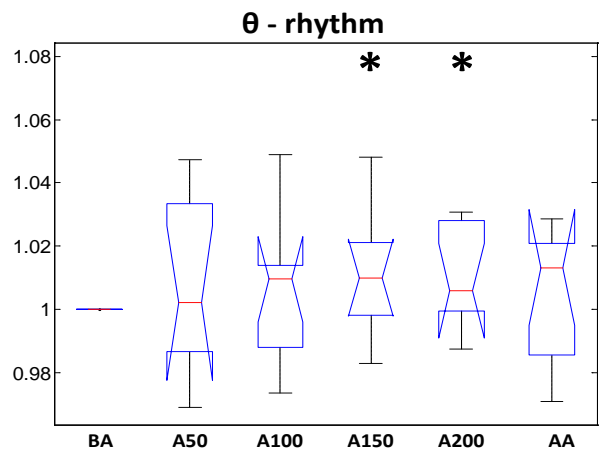

(c)

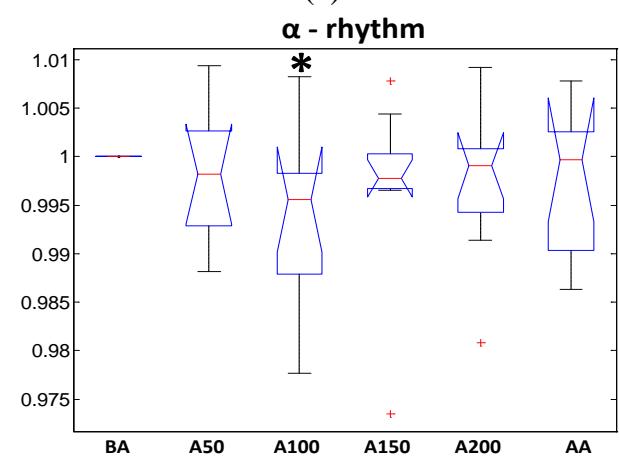

(d)

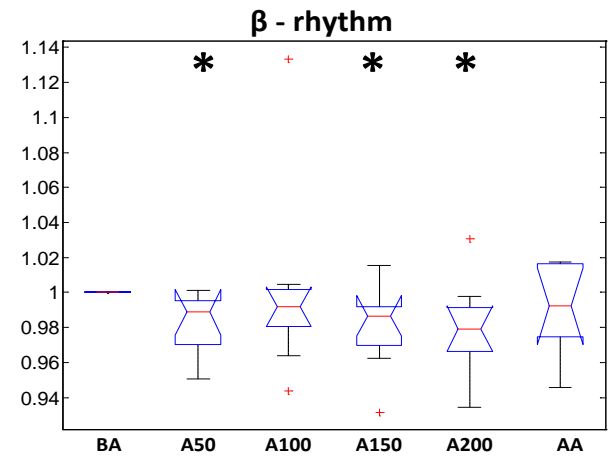

(e)

Fig. 3.Boxplot for normalized scaling exponent of averaged global brain domain under different acupunctureepochs at different EEG rhythm for nine subjects. (The star presents that there is different between current epoch and BA epoch at the level of 0.05.)

\section{CONCLUSIONS}

We use DFA method to study the EEG signals evoked by different acupuncture manipulations at acupointZusanli at different EEG rhythms from 9 subjects. It is found that a certain acupuncture manipulation has different effects on different EEG rhythms, and the response of a certain EEG rhythm to different acupuncturemanipulation is different. Moreover, we found that acupuncture induce the increase of scaling exponent at the delta and theta rhythm, while decrease at alpha and beta rhythm.

\section{REFERENCES}

[1] E. Ernst,A. R. White, "Prospective studies of the safety of acupuncture: a systematic review," The American Journal of Medicine, vol. 110, issue. 6, pp. 481-485, 2001.

[2] S. Knardahl, M. Elam, B. Olausson, "Sympathetic nerve activity after acupuncture in humans,” Pain, vol. 75, issue. 1, pp. 19-25, 1998.

[3] NIH, "NIH consensus development panel on acupuncture," J Am Med Assoc, vol. 280, pp.1518-1529, 1998

[4] P. A. Watters,F.A.Martin, "mthod for establishing long-range power law correlation from the electroencephalogram,"Biol Psyciatry, vol. 66, pp. 79-89, 2004.

[5] C. K. Peng,S. V.Buldyrev, S. Havlin S, "Mosaic organization of DNA nucleotides,” Physical Review E, vol. 49, issue. 2, pp. 1685-1689, 1994.

[6] S. Leistedt, M. Dumont, J.-P. Lanquart, "Characterization of the sleep EEG in acutely depressed men using detrended fluctuation analysis," Clinical neurophysiology, vol. 118, issue. 4, pp. 940-950, 2007.

[7] M. Jospin, P. Caminal, E. W. Jensen, "Detrended fluctuation analysis of EEG as a measure of depth of anesthesia,” Biomedical Engineering, IEEE Transactions on, vol. 54, issue. 5, pp.840-846, 2007.

[8] J.M. Lee, D.J. Kim, I.Y. Kim,“Nonlinear-analysis of human sleep EEG using detrended fluctuation analysis,” Medical engineering \& physics, vol. 26, issue. 9, pp. 773-776, 2004.

[9] J.S. Lee, B.H. Yang, J.H. Lee, "Detrended fluctuation analysis of resting EEG in depressed outpatients and healthy controls," Clinical Neurophysiology, vol. 118, issue. 11, pp. 2489-2496, 2007.

[10] Z. Li,Y.K. Zhang, "Quantifying fractal dynamics of groundwater systems with detrended fluctuation analysis,” Journal of hydrology, vol. 336, issue. 1, pp. 139-146, 2007.

[11] C. K. Peng, S. Havlin, H. E. Stanley, "Quantification of scaling exponents and crossover phenomena in nonstationary heartbeat time series,” Chaos, vol. 5, issue. 1, pp.82-87, 1995. 